\title{
Obesity induction during adolescence in ICR-CD1 mice causes CVD risk in the adulthood
}

\author{
G. C. Baccan ${ }^{1,2}$, L. E. Díaz ${ }^{2}$, O. Hernández ${ }^{3}$, A. Gheorghe ${ }^{2}$, F. Perez de Heredia ${ }^{2}$, J. Mujico ${ }^{2}$, \\ M. De La Fuente ${ }^{3}$ and A. Marcos ${ }^{2}$ \\ ${ }^{1}$ Department of Biofunction, Institute of Health Sciences, Federal University of Bahia (UFBA), Salvador, Brazil, \\ ${ }^{2}$ Department of Metabolism and Nutrition. Institute of Food Science, Technology and Nutrition (ICTAN), Spanish National \\ Research Council (CSIC), Madrid, Spain and ${ }^{3}$ Department of Physiology. School of Biology, Complutense University of \\ Madrid (UCM), Spain
}

Obesity is an epidemic health problem that is associated with other pathologies, including diabetes, cardiovascular diseases and cancer. Obesity during adolescence is a strong predictor of increased risk of severe obesity in adulthood ${ }^{(1)}$. Due to its genetic similarity with humans, mouse represents a valid animal model to study the impact of early obesity on obesity-related health parameters in adulthood. The aim of this study was to find out whether diet-induced obesity (14 weeks) may result in physiological alterations in ICR-CD1 mice. To this end, sixteen ICR female mice ( 8 weeks old, $n=15$ ) were fed ad libitum and divided into two groups according to their diets (Harlan Laboratories): 1) standard chow (control group, $n=8)$ and 2) a high-fat diet (60\% calories supported from fat; obese group, $n=7$ ). Food and water intakes as well as weight gain were measured twice a week. Systolic blood pressure was assessed at the onset and at the end of the experimental period. Also after the 14 week-experimental period, mice were sacrificed, visceral fat was removed and weighed, and blood was collected for analysis of plasma triglyceride levels (autoanalyzer ACCUTREND ROCHE) and plasma concentrations of insulin, leptin, adiponectin, soluble E-selectin, vascular cell adhesion molecules (sVCAM-1) and intercellular adhesion molecules (sICAM) (Luminex-100 IS, multiplex assay kits). The statistical analysis was performed using GraphPad Prism Software (Version 5.0, La Jolla, CA). Student's- $t$ test was used to compare data between groups. Statistical significance was set at $P<0.05$. Average body weights in the two groups were significantly different from week 5 on. According to our results (Table 1), the treatment with a high-fat diet for 14 weeks in ICR-CD1 mice was effective to obtain an early obesity model, which resulted in higher levels for several inflammatory biomarkers and an increased cardio-metabolic risk in adult animals.

Table 1. Body weight, visceral fat, blood pressure and inflammatory biomarkers

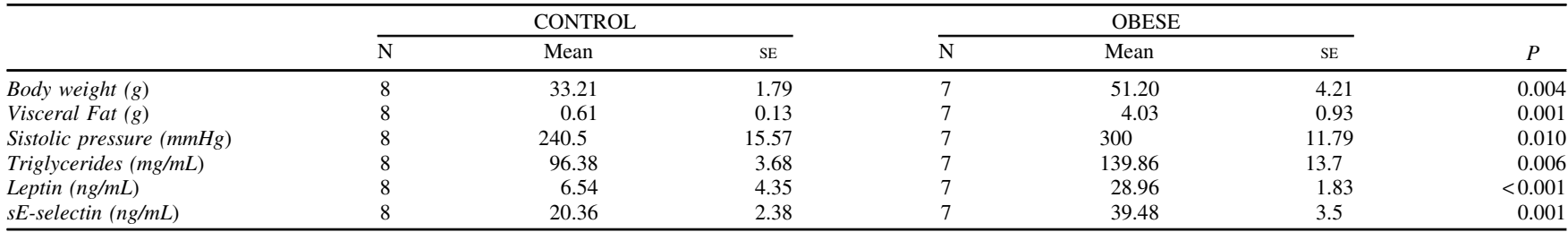

Support: This work was supported by MICINN (BFU2011-30336), UCM (910379ENEROINN) grants, RETICEF (RD06/0013/0003) from ISCIII and BTSA (The PRONAOS Study, CDTI 2008 1114).

1. Serdula MK, Ivery D, Coates RJ et al. (1993). Prev Med 22: 167-177. 